\title{
Marzanna: immagine soprannaturale della morte nella fraseologia slava
}

\begin{abstract}
L'esperienza della morte è un evento che per sua stessa natura va oltre la comprensione umana della realtà fisica ed è concepito come tipicamente soprannaturale; non può essere completamente spiegato con il pensiero razionale. In questo articolo analizzeremo la figura di Marzanna/Mara/Morena nel folclore e nella mitologia slava. La sua immagine è associata con le forze della morte, dell'inverno e i rituali di cambiamento stagionale. Questo complesso di riti e spiriti soprannaturali è studiato tra gli altri da Afanasyev e occupa una posizione rilevante nell'ambito dell'etnolinguistica. L'indagine su Marzanna è vista come strumento teorico per meglio comprendere differenti elementi fraseologici in varie lingue slave e la loro relazione con la rappresentazione della morte nel folclore europeo.
\end{abstract}

Parole chiave: fraseologia slava, mitologia comparata, folclore slavo, etnolinguistica, Marzanna, fraseologia della morte

\section{Introduzione}

In questo articolo analizzeremo come nel mondo slavo intorno alla figura mitologica e folclorica di Marzanna/Mara/Marena si possano identificare un insieme di campi semantici, accomunati dalla vicinanza al termine morte nelle varie lingue slave (pol. śmierć, ceco smrt, rus. смерть). Vari autori ipotizzano che la radice etimologica di Marzanna sia da collegarsi al protoindoeuropeo *mar/mor, che avvicinerebbe il concetto della morte all'idea delle acque e di condizioni liminali dell'anima, come incubi e angosce.

Secondo Łuczyński (Łuczyński, 2008), nel folclore slavo Marzanna era originariamente un personaggio mitologico, più specificatamente una dea dalle molteplici funzioni e attributi, relativi, tra le altre cose, alla morte. In un secondo momento divenne la personificazione stessa della morte e dell'inverno e infine, con il nome Marzanna, venne chiamato un pupazzo di paglia che, dopo essere stato portato in processione, veniva an- 
negato o distrutto durante riti ciclici di cambio stagionale. Sia la distruzione e in maggior misura l'annegamento del fantoccio, collegano Marzanna all'acqua e alle spaventevoli condizioni della mente, provocate dal sopraggiungere della morte. Alcuni aspetti del rito folclorico di Marzanna saranno utilizzati in questo articolo come strumento teorico per analizzare il contenuto dei dati fraseologici, idiomatici e verbali inerenti all'evento di natura sovrannaturale della morte nelle lingue slave e mostrare i legami semantici con i concetti di incubo, acqua, inverno e oscurità.

A tal proposito, inizieremo con il descrivere degli elementi principali del rituale. Successivamente vedremo le ipotesi riguardanti il proto lessema *mar/mor, dal quale i termini Marzanna/Mara/Morena derivano e le sue relazioni etimologiche con l'idea di morte, acqua e incubo notturno. Nell'ultima parte dell'articolo presenteremo alcuni elementi fraseologici e idiomatici relativi a Marzanna e al campo semantico della morte.

\section{Marzanna, Mara e Morena: personaggi soprannaturali nella mitologia e nel folclore slavo}

La mitologia protoslava pre-cristiana contiene al suo interno numerosi esempi di creature magiche ed eteree, divinità e spiriti, spesso associati ad elementi naturali. Nella visione del mondo degli antichi popoli slavi la realtà era composta da innumerevoli fenomeni non comprensibili solamente mediante i cinque sensi, ma che necessitavano di essere integrati in una comprensione del mondo che andasse oltre la realtà fisica, una concezione soprannaturale.

Nel folklore slavo, dopo secoli di cristianizzazione che investì non solo l'ambito religioso ma anche quello culturale, all'interno di canzoni, rituali, indovinelli, fiabe ebbe modo di conservarsi una "mitologia minore", perfettamente integrata nei costumi e nelle tradizioni popolari. I rituali popolari erano finalizzati ad armonizzare l'elemento umano e lo spazio antropizzato, con le forze soprannaturali dei luoghi e della natura più selvaggia.

Queste forze sono spesso state identificate come manifestazioni personificate di spiriti e dèi. L'evento della morte può essere visto come risultato di queste forze, come se una forza esterna agisse sulla persona (Meshcheryakova, Wysoczański, 2015: 427), un evento così pregnante di significato da andare oltre i limiti della realtà fisica, connotato trascendentalmente e posto al centro delle riflessioni religiose.

Indubbiamente la concettualizzazione della morte è mutata durante i secoli all'interno delle culture slave (e in particolar modo con l'avvento del cristianesimo), ma certamente ha mantenuto la sua centralità nei rituali religiosi e più generalmente nella visione linguistica del mondo propria di queste culture. In questo ambito la visione linguistica del mondo è un metodo di presentazione concettuale dell'universo che può essere ricostruito dai fatti linguistici.

La comune matrice mitologica slava ha portato alla coincidenza dell'immagine della morte in particolare nella cultura russa e nella cultura polacca (ibidem: 428) e, aggiungeremo noi, questa deduzione può essere estesa anche alle altre culture slave, portando ad avere una comune immagine della morte che sottenda l'intero gruppo linguistico. 
Seguendo l'esempio delle culture russa e polacca, tale immagine è considerata di natura femminile, similmente a molti popoli indoeuropei (ibidem: 425-426), per quanto non manchino anche rappresentazioni della stessa come appartenente al polo maschile. Nel pantheon protoslavo le divinità correlate ai riti popolari e stagionali della morte sono di natura femminile e conosciute in differenti areali come Marena/Mara/Marzanna (Petrukin, Agapkina, Vinogradova, 1995: 253).

Nei vari territori di insediamento dei popoli slavi troviamo diverse parole imparentate con la stessa forma. Nei territori slavi orientali la forma prevalente è connessa ai lessemi Marena/Mara, mentre in Cechia e Slovacchia con i lessemi Morena/Marmoriena. Nei territori polacchi la forma più comune è Marzanna, o una sua variazione.

Michał Łuczyński nell'articolo “La definizione cognitiva di Marzanna - un tentativo verso la ricostruzione di un frammento della visione del mondo tradizionale mitologica slava", definisce Marzanna come personificazione di un concetto astratto degli stereotipi di sfera religiosa (che sono presenti nei rituali folclorici) e al contempo come figura mitologica (Łuczyński, 2008: 173). Tale personificazione è rafforzata dal fatto che, in determinati contesti, Marzanna è coiponimo di termini strettamente legati al termine per definire la morte (pol. śmierć, rus. cmepmb) come pol. Śmiercicha, Śmiertusecka, Śmiertuska, Mór, ceco Smrt, Smrtnica, slovacco Šmertka (ibidem: 176; Agapkina, 2002: 601).

Le fonti utilizzate inerenti ai rituali di Marzanna coprono tutto il mondo slavo, in particolar modo i paesi slavi occidentali ed in minor misura quelli orientali (SDES, t. III: 179). Si tratta di lavori etnografici, raccolte e descrizioni di canzoni popolari e credenze religiose slave. I rituali qui esaminati sono parte di un calendario ciclico rurale che fa corrispondere ai cicli della natura necessari cicli rituali, che allineano simbolicamente l'interiorità dell'uomo ai manifesti cambiamenti esterni (minor o maggiore presenza di luce, nascita o morte della natura, ecc.).

I rituali di Marzanna sono legati al momento tra la fine dei mesi invernali e l'inizio della primavera. Seguendo il ciclo della natura, la loro esecuzione avveniva tra la fine di febbraio e l'inizio di marzo. Come conseguenza della cristianizzazione, si creò un sincretismo temporale situando il periodo del rito nella quarta o quinta domenica della Quaresima, in un giorno chiamato non a caso in slovacco Čierna, Smrtná, Marmurienová, nedel'a, e in ceco Smrtná nedéle (ibidem: 180). In questo caso il giorno del rito sovrappone nella sua denominazione i termini indicanti nero, morte, domenica, e si noti come sia la figura di Marzanna a dare il nome al periodo e non viceversa (Agapkina, 2002: 600). Il famoso cronista medievale polacco Jan Długosz riporta una tra le prime testimonianze scritte di Marzanna, databile al XVI secolo in Polonia, dove essa è indicata come divinità agricola, dea della fertilità, simboleggiata dal grano e dalla paglia, simile alla dea latina Keres/ Cerere (Petrukhin, 1995: 253).

Il fatto che la dea della fertilità sia anche legata alla morte è una contraddizione solamente a livello molto superficiale, in quanto nella visione del mondo antica la rigenerazione della terra e della vegetazione (da cui chiaramente il sopraggiungere della fertilità) poteva avvenire solamente come conseguenza di un ciclo perpetuo di morte e rinascita. Considerate le caratteristiche del presente articolo non sarà nostro compito scendere ad una dettagliata analisi della complessa relazione tra Marzanna, il suo ruolo come dea della 
fertilità e il calendario ciclico rurale. Tali questioni sono state approfondite da Łuczyński (2006) e Niedzielski (2011). Sarà nostra intenzione, invece, soffermarci sui significati semantici del rituale che possono essere associati con la morte. I resoconti del rituale giungono principalmente da Polonia, Slesia, Cechia, Moravia, Slovacchia, e Ucraina e sono contenuti nei lavori di Agapkina (2002: 600-606), degli autori di Slavyanskie Drevnosti (1995-2004: 180), Łuczyński (2006), e Niedzielski (2011).

L'analisi degli stessi mostra che, superate alcune differenze locali, vi sono differenti elementi comuni che delimitano il rituale di Marzanna. In esso possiamo osservare in sequenza:

- la creazione di una bambola o pupazzo di genere femminile realizzato in paglia e vestito di colori chiari (principalmente bianco),

- l'elevazione del pupazzo su un palo, portato così in processione attraverso il villaggio o la cittadina,

- la fine della processione fuori dall'area abitata e antropizzata e la distruzione del pupazzo mediante affogamento, incendio o sepoltura nella terra,

- l'introduzione di un piccolo albero o ceppo all'interno del villaggio.

Secondo il parere di Łuczyński (2008: 174) in uno primo stadio Marzanna/Mara era la divinità slava della fertilità e della morte e il pupazzo di paglia nominato in suo onore simboleggiava la morte e l'inverno. Successivamente è molto probabile che sia avvenuta una traslazione di significato dal nome della dea verso un'oggettivizzazione dei suoi attributi nel pupazzo. Il pupazzo di Marzanna divenne così esso stesso il simbolo della morte e dell'inverno, occultando l'originaria presenza "non materiale" della divinità alla quale faceva da tramite. Questa traslazione ha fatto sì che l'atto di estromissione di Marzanna dal villaggio rappresentasse l'allontanamento dell'inverno e della morte. Tale "esilio" veniva seguito dalla distruzione del pupazzo. Nonostante siano documentati vari metodi di distruzione di Marzanna, ci sembra essenziale qui sottolineare l'annegamento del pupazzo, in quanto diffuso in differenti areali - Slovacchia, Cechia, Moravia, Polonia (SDES, t. III: 180) ed in quanto indica una connessione diretta della morte e di Marzanna con l'elemento acquatico. Dunque, grazie a differenti metodi, con l'annientamento di Marzanna la morte e l'inverno erano espulsi dallo spazio antropico, in modo da propiziare la venuta della primavera e il ritorno della vita naturale.

La primavera e la nuova vita erano personificate in un giovane albero o ceppo di legno che era portato all'interno del villaggio dopo la distruzione di Marzanna. Il giovane albero era conosciuto sotto differenti nomi, tra i quali troviamo pol. gaj, drzewko, wiosna, nowe latko, slovacco zelený háj, leto. L'alberello simboleggiava non solo la primavera, ma il punto di svolta per un nuovo inizio e il preludio di un anno (pol. lata, forma plurale di "anni" come il rus. zoдa) (Agapkina, 2002: 605), e la stagione calda (pol. lato, rus. лето, "estate"). Mentre Marzanna veniva associata con il polo femminile, Gaj/Leto era associato con una figura maschile. La seconda parte del rituale, quella concernente il ritorno della vegetazione e della vita, non sarà qui maggiormente investigata ma si approfondirà la prima parte legata all'idea di morte, all'elemento dell'acqua e agli incubi. 


\section{Possibili sviluppi e campi semantici: la radice ipotetica*mar/ mor:}

Nei territori slavi, le figure mitologiche associate con il pupazzo usato nei rituali sopra descritti assumono molteplici nomi. Per essi è stata proposta una derivazione da una forma protoslava *Mara, -na $/ *$ Morana, -ena; ceco, croato, bulgaro, ucraino, bielorusso Mara; forme con il suffisso -na: pol. Marzan(n)a, ceco Mařana; ceco Mařena, slovacco Marejna, ucraino Marena; pol. dial. Morzana, ceco, slovaccco Morena / Mořena; pol. dial. (Slesia) Marzyna, ceco Mařina, slovacco Maryna, ucraino Maryna; slovacco Marmoriena, Marmuriena, Muriena (Łuczyński, 2008: 174). Ci pare di interesse notare come la versione slovacca del teonimo (Marmoriena, Marmuriena) appaia simile al nome del dio romano Marte, lat. Mars, chiamato anche Mamers, Mamercus, Mamurius in altre lingue italiche (Niedzielski, 2011: 170). Questo potrebbe indicare una primordiale funzione di entrambe le divinità in collegamento al mondo agricolo, dove forse arcaicamente Marte svolgeva il ruolo di protettore in armi delle attività rurali (Del Ponte, 1999: 150).

\subsection{Marzanna come morte: una derivazione dal protoindoeuropeo (PIE)}

Al fine di meglio comprendere la serie di lessemi relativi a Marzanna/Mara/Morena, sono state proposte differenti teorie da un'ipotetica radice nel PIE * mar/mor. Come riportato nel dizionario Slavyanskie Drevnosti tom III (1995-2004: 179-180) il termine Marzanna verrebbe dalla forma *mar che potrebbe essere una variazione di *mor, entrambe sono radicate nel protoslavo *sbmbrtb, similmente *mbrtb, letteralmente "morte", pol. śmierć, rus. смерть, ceco smrt, slovacco smrt'. Una simile radice è comune in differenti lingue europee antiche e contemporanee per esempio la si riscontra nei derivati sostantivi latino mors, lettone mirti, lituano mirtis. Una vicina radice PIE può essere indicata per il verbo "morire", in particolar modo di cause naturali, * $\mathrm{mr} / \mathrm{mer} / \mathrm{mir}$, da cui per esempio il latino mori (e l'italiano morire) rus. умереть pol. umrzeć e lituano mirti (Žujkova, 1998: 77).

\subsection{Incubi e condizioni liminali del corpo e della mente}

Il sopraggiungere della morte nelle credenze popolari è spesso rappresentato come l'avvicinamento di numerose creature soprannaturali che non solo provocano la morte, ma causano anche incubi, allucinazioni notturne, rovina e sfortuna. La condizione della morte è collegata all'assenza di movimento del corpo e tale immobilità è stata associata al sonno (Krzyżanowska, 1998: 105).

D'altro canto, una condizione di malattia grave, nella quale l'essere umano si trova tra la vita e la morte spesso porta ad alternare stati di veglia e stati di sonno con terribili incubi che possono essere correlati all'idea di morte. In questo modo è possibile ipotizzare un percorso di slittamento semantico mediante il quale la radice *mar si è sviluppata nelle lingue slave e in altre lingue europee verso un gamma di significati indicanti "spaventose visioni notturne che avvengono durante il sonno", originate da creature malvage e soprannaturali. Infatti, troviamo anche una forma protoslava * mara comune a tutte le lingue slave, con il significato di "fantasma, allucinazione" (SDES, t. III: 178). Un successivo 
sviluppo di questo termine si trova nell'ucraino мapa che indica un non meglio specificato spirito maligno, che oscura gli occhi dirigendo i malcapitati in luoghi pericolosi (ibidem). In molteplici occasioni l'idea della morte è assimilata all'idea di un viaggio, di una partenza.

Si potrebbe argomentare come simili luoghi pericolosi, verso i quali мapa conduce, siano collegati alla morte perché nel folclore ucraino мapa è solita spiare dalle finestre i dormienti di notte e in tal modo rovinare perdutamente la salute delle persone, alcune volte causandone la morte. All' inizio del XIX secolo in slovacco Morena era identificata come morova žena lett. "la signora della pestilenza" e il rituale precedentemente menzionato era anche messo in atto per proteggere gli esseri umani e il bestiame dalle malattie che essa mandava (Agapkina, 2002: 603).

Come scrive Alexander Afanasyev, rus. мapa, pol. mara, zmora sarebbe uno spirito demoniaco di un morto o morente che perseguita le persone (e che si può nutrire con il sangue come il popolare vampiro) (Afanasyev, 1869: 35). Lo scrittore traccia parallelismi con i termini ingl. nightmare, ted. nachtmar, franc. cauchemare (e da esso pol. koszmar rus. кошмар). Nel folclore russo мара appare anche complementare ad un'altra creatura magica e demonica chiamata кикимора.

\subsection{0monimia nell'ipotetiche radici per "morte" $e$ "mare"}

La radice indoeuropea * mar/mor non è solamente associata all'idea di morte ma anche con l'idea di “qualcosa di collegato all'acqua” (Luczyński, 2006: 172) come il pol. morze, ted. meer, lat. mare, rus. мope, lett. "mare". Un'altra etimologia che lega *mar/mor con le acque è il dialettale slesiano maras, rus. мapa indicante "palude, acqua stagnante" (Vlasova, 2008: 123-124). Il mare e, più in generale le distese di acqua profonde, sono collegate con l'aldilà e la terra dei morti (Słownik stereotypów i symboli ludowych, 1999: 393).

Così la stessa ipotetica radice per il teonimo di Marzanna è indicata come omonimo con lessemi legati all'acqua e ad elementi soprannaturali acquatici, come il casciubo moronka "pescatrice" rus. моряны "sirenette" (Luczyński, 2008: 175), e l'italico Marica, dea o ninfa delle acque (Del Ponte, 1999: 170). Alexander Famincyn (1884: 403) riporta in Bozhestva Drevnikh Slavyan, che la similarità di funzioni tra Marzanna e Marica e altri personaggi mitologici di deità femminili come variazioni della forma Marica/ Maria, Маренаa possono essere trovati in canzoni popolari dalle sponde dell'Adriatico fino al Mar Baltico.

\subsection{Inverno e oscurità}

Nell'opera Poeticheskiye vozzreniya slavyan na prirodu, Afanasyev (1869: 35) prende in considerazione anche altri concetti che si trovano nello stesso campo semantico della morte, e che con esso mantengono rapporti etimologici: oscurità e inverno (rus. мрак, pol. mrok, e rus. зимa, pol. zima). Per quanto riguarda l'oscurità e la morte è una caratteristica comune a molte culture associare la vita con la luce del giorno e la morte con l'oscurità e il buio (Krzyżanowska, 1998: 104). L'aumentare progressivo di oscurità e freddo in inverno permette una vicinanza semantica della morte con il buio e il gelo, come in ucr., bulg., rus. мороз, slovacco mráz, pol. mróz. Nella cultura russa la morte è assimilata 
al gelo, a causa della sua potenza ineluttabile, essendo il gelo causa fondamentale di morte in inverno (Meshcheryakova, Wysoczański, 2015: 426).

Secondo l'opinione di Grzegorz Niedzielski (2011: 172), la vicinanza tra il gelo invernale e la morte è relativa alla morte della terra in opposizione con il concetto di natura rigogliosa e viva. In inverno il suolo è coperto dalla neve e dal ghiaccio, e in qualche modo "scompare" alla vista, è assente. Se si considera la morte come assenza di vita e l'inverno come scomparsa o temporanea assenza della terra si può evidenziare un'opposizione tra inverno/terra simile all'opposizione morte/vita. Questa opposizione si manifesta nella vicinanza tra le parole per "inverno" e "terra", ad esempio in pol. ziemia/zima e latino hiems/humus (Niedzielski, 2011: 172).

\section{Elementi fraseologici: Marzanna come personificazione della morte e campo semantico relativo alla morte nelle lingue slave}

In questo paragrafo del nostro articolo inizialmente riporteremo differenti elementi fraseologici che si riferiscono a Marzanna come spirito malvagio, alla sua influenza malefica, e di conseguenza di Marzanna come sinonimo di morte e malattia. Successivamente riporteremo altri elementi idiomatici che sono inclusi nello stesso campo semantico (inverno e oscurità) e metafore del concetto di morte: sonno come morte, morte come andare verso le acque.

\subsection{Mara/Marzanna come spirito malvagio e la sua influenza ingannatrice}

Ucr. Ходить як мара - essere confusi (SDES, t. III: 178-179).

Ucr. Блудить як якась мара - essere confusi (ibidem).

Rus. Мара водит кого-то - lett. "Mara conduce qualcuno", agire in modo strano o inusuale (Mokienko, 2007: 384).

Rus. Не будешь прясть, так Мара придёт - lett. “(Se) Non filerai, allora Mara arriverà" (SDES, t. III: 178-179).

In questa forma di avvertimento l'attività tipica delle donne nelle società contadine, il filare, potrebbe essere avvicinato al filare delle creature mitologiche dette Moire o Parche che nella mitologica greca e romana filavano e tessevano i destini e la vita degli uomini. Una volta che il loro filare finiva, sopraggiungeva la morte.

Rus. Вон Мара придет тебя заберёm - lett. "Ecco Mara arriverà e ti porterà via" (detta specialmente per spaventare i bambini) (ibidem).

Slovacco Mara bi ce uźala! - lett. "Che Mara ti prenda!" (ibidem).

\subsection{Marzanna come sinonimo per morte e malattia}

Ceco Proti Mořeně není kořeně - Contro Morena non ci sono rimedi, sottolinenando l'inevitabilità della morte (SDES, t. III: 178-179).

Pol. Wynieśliśmy mór ze wsi - let. "Abbiamo portato la pestilenza fuori dal villaggio" in riferimento alla processione con il pupazzo di Marzanna (ibidem: 180). 
Ceco Pi̊jdeš na morán - Presto morirai (Afanasyev, 1994: 35).

Ceco Mořena na něj sáhla - lett. "È stato toccato dalla morte" (ibidem).

Ceco e Slovacco Smrt’Mařeně, a nám ni! Utikejte smrt nás honi! Smert z města, léto do města! - lett. "Morte a Marena e non a noi! Correte, la morte ci sta per prendere! (La) Morte (sia) fuori dalla città, (venga l') estate in città!" (SDES, t. III: 180).

Pol. Śmircicha ze wsi, nowe lotko do wsi - lett. "(Vada la) Morte fuori dal villaggio, (entri) il nuovo anno nel villaggio" (Agapkina 2002: 608).

Ceco Morena Morena za koho umerta? - lett. "Morena Morena per chi sei morta?" (SDES, t. III: 181).

Slovacco Muriena, Muriena za kohos'umerla? Za starého dedku, čo má bradu rie$d k u$ - lett. "Morena Morena per chi sei morta? Per l'anziano signore con la barba rada", qui l'espressione ha carattere apotropaico e la funzione di salvare un uomo anziano dalla morte (Agapkina, 2002: 603).

Slovacco Vynesli sme Morenu zo vsi, priniesli sme máj nový do si - lett. "Abbiamo portato Morena fuori dal villaggio, abbiamo portato dentro il villaggio il nuovo maggio" (ibidem: 606).

Pol. Leżé́ (spoczać) na marach - essere morto. In questa e nella seguente unità la parola mary significa "catafalco per i deceduti, sul quale è deposta la bara con il corpo" (Profantová, 1998: 113).

Pol. Widzieć kogoś na marach - vedere qualcuno morto (Skorupka, 1967: 424).

\subsection{Morte e sonno}

Dato che lo spirito malvagio conosciuto come Mara/Marzanna appare maggiormente durante la fase di sonno è importante esaminare gli elementi fraseologici che collegano la morte (della quale Marzanna in altri idiomatismi è considerata la personificazione) e il sonno in ambito più generale.

Pol. Zasnać snem głębokim - lett. "addormentarsi di un sonno profondo", morire (Krzyżanowska, 1998: 105).

Pol. Spać na wieki - “dormire per sempre”, morire (ibidem). In questa e nella successiva unità il termine "na wieki" e il russo "на веки веков" appaiono un calco slavo del latino medievale cristiano "in sæcula sæculorum", per indicare una cosa che si trascina a tempo indeterminato e di cui non se ne prevede il termine, da cui poi il significato di "per sempre".

Rus. Кто-то уснул на веки веков - “Qualcuno si è addormentato per sempre”, morire (Piasecka, 2011: 98).

Rus. Закрыл глаза - lett. "Ha chiuso gli occhi”, morire (ibidem).

Rus. Заснуть вечным (последним, мертвымм) сном - "Addormentarsi di un sonno eterno (finale, mortale)", morire (ibidem).

Rus. Смежить глаза (очи) - lett. "Chiudere gli occhi”, morire (ibidem).

Rus. Заснуть (почить, уснуть) вечнылм - lett. "Andare a dormire (riposare, addormentarsi) eternamente", morire (ibidem). 


\subsection{Morte e acqua}

Pol. Za morzem - lett. "Oltre il mare", con il significato di inferi, oltretomba. Si ricordi come nella mitologia classica il passaggio verso gli inferi implichi un tragitto oltre le acque del fiume Acheronte, usato anche come metafora per "morte" (Niedzielski, 2011: 171).

Pol. Na św.Grzegorza (12 marca) idzie Zima do morza - lett. "Nel giorno di Santo Gregorio, il 12 marzo, l'Inverno va nel mare" (ibidem: 170).

In questo modo di dire il mare è usato come metafora dell'oltretomba, e l'Inverno (personificato), sta morendo proprio nel periodo di esecuzione del rituale di Marzanna. In differenti varianti del rituale infatti il pupazzo è fatto annegare e dunque "va verso le acque".

L'aldilà e gli inferi sono anche associati (per esempio nella cultura latina e greca e in quella slava) con il luogo dove risiedono gli antenati, spesso situato in una zona sotterranea, o sotto profonde acque, dunque "morire" può essere espresso anche come:

Pol. Przenieść się do krainy swoich przodków - lett. "Portarsi verso la terra dei propri antenati”, morire (Krzyżanowska, 1998: 99).

Rus. Отправиться к праотиам - lett. "Essere mandato verso gli antenati", morire (ibidem).

\subsection{Inverno e oscurità}

Ceco Pohřbívají zimu - lett. "Abbiamo seppellito l'inverno", chiaro è il collegamento con il rituale di Marzanna, dove il pupazzo simboleggiante l'inverno e la morte veniva distrutto e in alcuni casi seppellito (Agapkina, 2002: 604).

Pol. Kraina nocy - lett. "Paese della notte" con il significato di aldilà, la terra dove non vi è luce, associata dunque con l'oscurità e la notte (Krzyżanowska, 1998: 104).

Pol. Ktoś odchodzi w mrok śmierci - lett. "Qualcuno sta andando nelle tenebre della morte", morire (ibidem).

Rus. Морочить - lett. "Imbrogliare", con il significato di creare oscurità nella mente di qualcuno (Molotkov, 1987: 254).

Rus. Не морочь мне голову - lett. "Non fare oscurità nella mia mente", con il significato di "non confondermi" (ibidem).

Rus. Уйти в мраки - lett. "Allontanarsi nell'oscurità", andare in malora, morire. (Mokienko, 2007: 415).

Rus. Наводить мороку - lett. "Condurre nell'oscurità (confusione)", con il significato imbrogliare, ingannare (ibidem: 412).

\section{Conclusione}

In questo articolo abbiamo dimostrato come la figura mitologica di Marzanna/Mara sia collegata a differenti elementi fraseologici, che associano la sua immagine al concetto di morte. In primo luogo, abbiamo analizzato Mara/Morena/Marzanna come divinità protoslava, le sue funzioni nel folclore popolare e mostrato la trasposizione di Marzanna da creatura soprannaturale a nome di un pupazzo femminile simboleggiante morte 
e inverno. In secondo luogo, abbiamo trattato le caratteristiche del rituale ciclico stagionale durante il quale il pupazzo viene distrutto. Successivamente abbiamo affrontato in dettaglio l'ipotesi riguardante la radice etimologica del lessema * mar/mor e mostrato la sua analogia con Marzanna e il termine protoslavo *sbmbrtb - morte. Infine, abbiamo elencato e commentato differenti elementi fraseologici e idiomatici in varie lingue slave, nei quali Marzanna è direttamente enunciata o che presentano legami semantici con la morte e il relativo campo semantico. La ricerca di ulteriori relazioni tra mitologia, elementi fraseologici e campi semantici può portare alla luce collegamenti tra lingue e sistemi mitologici che sottendono ad una visione del mondo linguistico-culturale arcaica pan-europea. Tale visione del mondo può essere tracciata nel folclore ed espressa nel mezzo linguistico.

\section{Literatura}

Агапкина Т.А. (2002), Мифопоэтические основы славянского народного календаря. Весенне-летний ичик, Часть ІІІ Формирование календарного символизма, Издательство Индрик, Москва.

Афанасьев А.Н. (1994), Поэтические воззрения славян на природу, (1865-1869), т. III, Издательство Индрик, Москва.

Власова М.Н. (2008), Энщиклопедия русских суеверий = Новая абевега русских суеверий = Русские суеверия: Энциклопедический словарь, Азбука-классика, Санкт-Петербург.

Мокиенко В.М., Никитина Т.Г. (2007), Большой словарь русских поговорок. Более 40000 образных выражений, ОЛМА Медиа Групп, Москва.

Молотков А., Сост. Л.А. Войнова, В.П. Жуков, А.И. Молотков, А.И. Федоров (ред.) (1987), Фразеологический словарь русского языка: свыше 4000 словарных статей, Русский Язык, Москва.

Петрухин В.Я., Агапкина Т.А., Виноградова Л.Н., Толстая С.М. (ред.) (1995), Славянская мифология. Энциклопедический словарь, Эллис Лак, Москва.

Толстой Н.И. et al. (1995-2004), СДЭС - Славянские древности. Этнолингвистический словарь, том 1-3, Институт славяноведения РАН, Москва.

Фаминцын А. (2014): Божества древних славян, (1884), Институт русской цивилизации, Москва.

\section{Bibliografia dopo trascrizione}

Afanasyev A.N. (1994), Poeticheskije vozzrenije slavian na prirodu, (1865-1869), t. III, Izdatelstvo Indrik, Moskva.

Agapkina T.A. (2002), Mifologicheskije osnowy slavyanskogo narodnogo kalendaria. Vesennie-letnij cikl, Chast III Formirovanije kalendarnogo simvolizma, Izdatelstvo Indrik, Moskva.

Del Ponte R. (1999), Dei e miti italici, Ecig, Genoa.

Famincyn A. (2014), Bozhestva drevnikh slavian, (1884), Institut russkoj civilizacii, Moskva. 
Krzyżanowska A. (1998), Ostatnia podróż - czyli polska i francuska metaforyka śmierci, „Etnolingwistyka: problemy języka i kultury”, 9/10, p. 93-109.

Łuczyński M. (2000), Semantyka obrzędów wiosennych zwiąanych z Marzanna i Jaryla, „Respectus Philologicus”, 10, 15, p. 165-176.

Łuczyński M. (2008), Kognitywna definicja Marzanny - Próba rekonstrukcji fragmentu tradycyjnego mitologicznego obrazu świata Stowian, „Studia Mythologica Slavica”, 11, p. 173-196.

Meshcheryakova O.A., Wysoczański W. (2015), Verbal Objectification of the Concept of Death in the Polish and Russian, "Mediterranean Journal of Social Sciences", 6: p. 423-429.

Mokienko V.M., Nikitina T.G. (2007), Bolshoj slovar russkikh pogovorok. Boleye 40000 obraznykh vyrazhenij, OLMA Media Grupp, Moskva.

Molotkov A. (red.), Sost. L.A. Voynova, V.P. Zhukov, A.I. Molotkov, A.I. Fe Dorov (1987), Frazeologicheskij slovar russkogo jazyka: svyshe 4000 slovarnykh statej, Russkij Jazyk, Moskva.

Niedzielski G. (2011), Królowie z gwiazd. Mitologia plemion prapolskich. Armoryka, Sandomierz.

Petrukhin V.Ya., Agapkina T.A., Vinogradova L.N. Tolstaya S.M. (red.) (1995), Slavianskaya mifologija. Enciklopedicheskij slovar, Ellis Lak, Moskva.

Piasecka A. (2011), Koncept śmierci w rosyjskiej frazeologii, „Folia Linguistica Rossica”, 7, p. 95-102.

Profantová, Z. (1998), Językowy obraz śmierci na Słowacji, „Etnolingwistyka: problemy języka i kultury", 9/10, p. 111-120.

Słownik stereotypów i symboli ludowych (1999), J. Bartmiński (red.), t. 1**, UMCS, Lublin.

Słownik frazeologiczny języka polskiego (1967), S. Skorupka, t. 1, Wiedza Powszechna, Warszawa.

Tolstoy N.I. et al (1995-2004): SDES - Slavianskije drevnosti. Etnolingvisticheskij slovar, tom 1-3, Institut slavianovedenija RAN, Moskva.

Vlasova M.N. (2008), Enciklopedija russkikh suyeverij = Novaja abevega russkikh suyeverij = Russkije suyeverija: Enciklopedicheskij slovar, Azbuka-klassika, St Petersburg.

Žujkova M. (1998), Nominacija smerti i arkhaicheskoje mysljenije, „Etnolingwistyka: problemy języka i kultury", 9-10, p. 67-80.

\section{Abstract}

\section{Marzanna: supernatural figure of death in Slavic phraseology}

Experience of death is an event that by its own nature is beyond the human understanding of physical reality, and it is seen as a supernatural one, which cannot be fully explained by rational thought. In this article, I will investigate the figure of Marzanna/Mara/Morena, in Slavic folklore and popular mythology. Her figure it is associated with forces of the death and seasons chancing rituals. This complex of rites and supernatural spirits is discussed by Afanasyev, and it has as a relevant position in the ethnolinguistics. The investigation of Marzanna is seen as a theoretical tool in order to better understand phraseology of Slavic languages and his relationship with pan-European folklore's depiction of death.

Keywords: Slavic phraseology, comparative mythology, Slavic folklore, ethnolinguistics, Marzanna, phraseology of death 


\section{Streszczenie \\ Marzanna: nadprzyrodzony obraz śmierci we frazeologii słowiańskiej}

Pojęcie śmierci to wydarzenie, które ze względu na swą naturę znajduje się poza granicami ludzkiej fizycznej realności i można je rozpatrywać jako zjawisko nadnaturalne, nieudające się racjonalnie wytłumaczyć. W artykule poddano analizie obraz Marzanny/Mary/Moreny w słowiańskim folklorze i mitologii. Jej obraz kojarzył się z pojęciem śmierci i obrzędami kalendarza ludowego opisanymi przez Afanasjewa. Podobne rytuały oraz istoty nadnaturalne są opisane w słowniku Starożytności słowiańskie i odgrywają ważną rolę w etnolingwistyce. Fenomen Marzanny rozpatrywany jest jako instrument teoretyczny do lepszego rozumienia frazeologii języków słowiańskich oraz jego związków z ogólnoeuropejskim folklorystycznym przedstawieniem śmierci.

Słowa kluczowe: frazeologia słowiańska, mitologia porównawcza, folklor słowiański, etnolingwistyka, Marzanna, frazeologia śmierci 